\title{
28 Research Square \\ RNA-seq analysis of circRNA profiles in the lens of Crybb2 knockout mice
}

\author{
Qian Gao \\ Wusong Central Hospital \\ Hao Xu \\ Changhai Hospital

\section{Yuping Duan} \\ Wusong Central Hospital

\section{Leiming Cai} \\ Wusong Central hospital

\section{Zhiwen Nie} \\ Wusong Central Hospital \\ Yaru Zou \\ Xi'an Central Hospital
}

Qian Li ( $\sim$ aqianmerry@163.com )

Wusong Central Hospital https://orcid.org/0000-0002-1586-8975

\section{Research Article}

Keywords: Circular RNA, Lens, Crybb2, RNAseq, Cataract

Posted Date: June 22nd, 2021

DOI: https://doi.org/10.21203/rs.3.rs-222281/v1

License: (c) (i) This work is licensed under a Creative Commons Attribution 4.0 International License.

Read Full License 


\section{Abstract}

Background: Currently, there is little information on the expression profiles of circRNAs in the lens. $\beta B 2-$ crystallin (CRYBB2) is an abundant protein in the mammalian lens, and its abnormal expression contributes to the development of cataract. This study aimed at exploring how Crybb2 knockout could modulate the expression profiles of circRNAs in mouse lens.

Methods and Results: We extracted total RNAs from the lens of wide-type (WT) and Crybb2 -/- mice and after depleted their rRNAs and broken the remaining RNAs, we reversely transcribed the RNAs into cDNAand sequenced them. Furthermore, we performed bioinformatics to identify and analyze the differentially expressed circRNAs and predicted their potential functions. We validated some differentially expressed circRNAs by quantitative RT-PCR. We employed RNA-seq to identify 49,494 circRNAs and compared to the WT lens, 149 circRNAs were upregulated and 172 downregulated in Crybb2 - $/$ - mouse lens. With the top 300 miRNA-circRNA interaction pairs, we constructed a network of circRNA-miRNA interactions. Moreover, those differentially expressed circRNAs participated in various biological processes, such as lens fiber cell development, calcium channel complex, structural constituent of the lens. They were involved in several important pathways, such as the canonical Wnt signaling pathway. Quantitative RT-PCR validated some differently expressed circRNAs in the lens of Crybb2 -/- mice.

Conclusions: Crybb2 knockout significantly modulated circRNA expression profiles in the lens of mice, which may help clarify the roles of circRNAs in age-related cataracts.

\section{Introduction}

A cataract is characterized by the clouding in the lens of the eye, and the leading cause of visual impairment and blindness worldwide. However, the etiology and pathogenesis of the disease remain unclear. Cataracts are attributed to abnormal aggregation of proteins in the lens, which scatters and interferes its accommodation for the light focusing onto the retina. There are three major soluble crystallins, $\alpha-, \beta$-, and $\gamma$ and $\beta B 2$-crystallin (CRYBB2) is the most abundant one among $\beta$-crystallins. The CRYBB2 can interact with other $\beta$-crystallins to form hetero-oligomers and is crucial for the transparency of the lens. Evidently, Crybb2/- spontaneously develop cataracts and their severities increase with age[12]. However, how CRYBB2 expression is regulated in the lens has not been completely understood.

Circular RNA (circRNA), a kind of non-coding RNA, is widely transcribed in eukaryotic cells. Structurally, circRNA has a closed loop, and has the unique characteristics of huge varieties, stable structure without sensitive to RNA exonuclease, conservative sequence and specific expression in cells or tissues. CircRNA is produced by alternative splicing of a specific precursor mRNA in a manner of exon or intron cyclization. Functionally, circRNAs can mainly cis-regulate their parental gene expression[3], regulate the stability of some proteins by binding to them[4], sponge and sequester specific miRNAs from their targeted mRNAs[5]. Actually, circRNAs can regulate the pathogenesis of various diseases, including nervous system diseases and tumors[6-7]. Currently, there are few reports on the role of circRNAs in the 
development of age-related cataract. CircRNA may affect the function of lens epithelial cells by regulating mir-184, which leads to the development of ocular diseases[8]. Furthermore circHIPK3 silencing upregulates miR-193a expression to increase the apoptosis of human lens epithelial cells by downregulating CRYAA expression[9]. However, how CRYBB2 regulates the expression of circRNAs in the lens has not been explored.

To address these questions, we characterized the differentially expressed circRNA profiles in $C r y b b 2^{\prime-}$ mouse lens by RNA-Seq analysis of rRNA-depleted RNAs. We further performed bioinformatics to determine the exon number and length distribution of the circRNAs in the lens. After identified differentially expressed circRNAs, we analyzed the circRNA expression patterns and their potential functions by Gene Ontology (GO) annotation and Kyoto Encyclopedia of Genes and Genomes (KEGG). Finally, we validated the expression of representative differentially expressed circRNAs in wide-type (WT) and $\mathrm{Crybb2}^{--}(\mathrm{KO})$ mouse lens by quantitative RT-PCR. Our data indicated that $\mathrm{Crybb2}$ knockout significantly changed the expression profiles of circRNAs, which may contribute to the development of cataract in mice.

\section{Materials And Methods}

\section{Animals and tissue collection}

This study was granted by the Animal Care and Use Committee of Shanghai General Hospital (Shanghai, China). We used wide-type (WT) C57BL/6 mice (both male and female, the Experimental Animal Center of the Second Military Medical University, Shanghai, China). We obtained $C r y b b 2^{-1-}$ mice from the Ingenious Targeting Laboratory (Ronkonkoma, NY, USA)[1]. We maintained the mice in a pathogen-free room with 12-h light/dark cycles and free access to food and water ad libitum. The study was performed in accordance with the ethical standards of the 1964 Declaration of Helsinki and later amendments. This study was conducted in accordance with institutional guidelines and approved by the Animal Care and Use Committee, Wusong Center Hospital (permission number 2020-Y-10).

We anesthetized six months old WT and $C r y b b 2^{-/}$mice ( $\mathrm{n}=3$ per group) with pentobarbitone $(150 \mathrm{mg} / \mathrm{kg})$, dissected their lens, immediately frozen them in liquid nitrogen and kept at $-80^{\circ} \mathrm{C}$.

\section{RNA extraction and RNA-Seq}

We extracted total RNAs from the samples and digested ribosomal RNA using the ribo-zero kit (IIlumia囚 Cat.No.RS-122-2301). After determined the RNA concentrations using the NanoDrop ND-2000 (ThermoFisher Scientific, Waltham, MA, USA), we generated a RNA library and broke the circRNA into short fragments. RNA-seq library preparation was carried out using the NEBNext $\circledast$ Ultra ${ }^{\text {TM }}$ Directional RNA Library Prep Kit for Illumina.Subsequently, we reversely transcribed the RNA fragments into cDNA and evaluated its quality in the Agilent 2100 Bioanalyzer. Finally, we sequenced the cDNA library in an Illumina HiSeqTM 2500 system following the vendor's recommended protocol (TruSeqR RNA Sample 
Preparation v2 Guide, Illumina Company Ltd). HTSeq software was used to count the reads numbers mapped to each circRNA.

\section{CircRNA sequencing analysis}

We used the NGSQC Tool kit (version number v2.3.3) to evaluate the quality control and removed the lowquality reads to obtain high-quality clean reads. Simultaneously, we used the FASTQC2 (version number v0.10.1) software to evaluate the data quality. Next, we employed the BWA MEM algorithm (version 0.7.5a) to align clean data against the reference gene sequence and detected circRNA using the CIRI software. According the circRNA position and the protein-coding gene annotation in the database, we obtained the sequences and counted the number of circRNA in each sample. We statistically analyzed the circRNAs for their length distribution, the chromosome distribution, and the number of circRNA exons. Data are expressed as RPM4 (reads per million mapping) in box plots and Venn diagrams using online tools (www.omicshare.com/tools/Home/Soft/box and www.omicshare.com/tools/Home/Soft/venn).

\section{Unsupervised hierarchical clustering and PCA}

We employed the Deseq25 package to perform principal component analysis (PCA), and investigate the distribution and relationship between the samples. In addition, we clustered the samples to investigate their similarity. We also performed unsupervised hierarchical clustering analyses using the gmodels in $\mathrm{R}$ (https://cran.r-project.org/web/packages/gmodels/index.html) and PCA using the ggplot2 in R (https://cran.r-project.org/web/packages/ggplot2/index.html) [10] .

\section{GO and KEGG pathway analyses}

$\mathrm{GO}$ annotations are useful for predicting the functions of gene products across numerous species. The GO categories were derived from the GO database. KEGG is a main public pathway-related database. The KEGG database is used to perform Pathway analysis of genes from different circRNA sources. We estimated the molecular function (MF), biological process (BP) and cellular component (CC) of differentially expressed circRNAs by GO analysis and predicted the functional enrichment of differentially expressed circRNAs by the KEGG pathway analysis. We determined the differentially expressed circRNAs using the edgeR package, based on $\mathrm{FC} \geq 2$ and $\mathrm{p}<0.05$, and generated volcano plots using the ggplot 2 in R.

\section{Prediction of circRNA-miRNA interactions}

As circRNAs were shown to have an impact on miRNA-mediated regulation of gene expression through miRNA sequestration\we constructed a network of differentially expressed circRNAs using the TargetScan and Miranda.We selected the high confidence miRNAs in humans. Hypergeometric distribution test was used to identify the miRNAs that have greater influence in the differential circRNA. The calculated result returned an enrichment significance $p$ value. The small $p$ value indicated that the differential circRNA was enriched in the miRNA. For the enrichment results of total differential circRNA, 
we sorted by $p$ value, top 300 miRNA-circRNA interaction pairs with smaller $p$ value were extracted, and predicted a circRNA-miRNA regulation network using the Cytoscape (V3.2.0).

\section{Quantitative real-time polymerase chain reaction (qRT-PCR)}

We extracted total RNA from the lens using TRIzol reagent (Invitrogen) and reversely transcribed each sample into cDNA using the RevertAid First Stand cDNA Synthesis Kit (GeneCopoeia, Cat AORT-0020). The relative levels of each differentially expressed circRNA transcript to the control GAPDH were determined by qRT-PCR in the LightCycler480 $\otimes$ Real-time PCR Instrument (Roche, Swiss) using the QuantiFast SYBR Green PCR Master Mix (Qiagen, Germany) and specific primers. We performed the PCR reactions in triplicate as the following protocol: $95^{\circ} \mathrm{C}$ for $5 \mathrm{~min}$, and 40 cycles of $95^{\circ} \mathrm{C}$ for $10 \mathrm{~s}, 60^{\circ} \mathrm{C}$ for $30 \mathrm{~s}$. The primer sequences were circRNA_03118: Forward 5'-GTCTTCTTTGGTGGAACCTACA-3', Reverse 5'-TTGGAGAAATGGTGCTTTGC-3'; circRNA_05302: Forward 5'-CCACATGGAGACAGCAGA-3' Reverse 5'-GATAAGCCACTTCGTCCC-3';

circRNA_09966: Forward 5'- CATAGCAGGAGTGGAGAG-3' Reverse 5'-AAACAGCTACAGGAAACCTT3'; circRNA_10276: Forward 5'-GTGAGCCCTGGCTTTGA-3', Reverse 5'-TACTTTGTCCTGTTTGGCTG-3';

circRNA_14121: Forward 5'-CATCCGGAGACCCATACTG-3', Reverse5'-GGATCAAACACTTCTGAAGCCA-3'; circRNA_16505: Forward5'-CCACAACAACAGGAAACG-3', Reverse 5'-GCTGGTGTAACACTGAAGAT-3'; circRNA_34578: Forward 5'-CTGGCACTTCAAGTCTTCAT-3', Reverse 5'-AAGCCAAGAGCTGCTTTAG-3'; circRNA_36601: Forward 5'-GCTACGAGCAGGCTAATTG-3', Reverse 5'-CTGCGTCCCACTTTGATG-3'; GAPDH: Forward 5'-TCATCCCAGAGCTGAACG-3', Reverse 5'-TCATACTTGGCAGGTTTCTCC-3'. The data were analyzed by the $2^{-\triangle \triangle C t}$ method (Livak and Schmittgen, 2001).

\section{Statistical analysis}

The data were analyzed by Student's t-test using R software version 3.2.1(http://www.r-project.org/). A two-side P-value of $<0.05$ was considered statistically significant.

\section{Data access}

RNA-seq data were submitted to Sequence Read Archive (SRA) under accession number PRJNA728889 (https://www.ncbi.nlm.nih.gov/sra/PRJNA728889).

\section{Results}

\section{CircRNA characteristics in mouse lens}

We used the RNA-seq technology and the CIRI software to analyze circRNA profiles in mouse lens. Based on the available data, we predicted 20894, 27697 and 22876 circRNAs in the lens from three $\mathrm{Crybb2}^{-1-}$ mice and there were 3083,5627 and 3287 unique circRNAs in those mice, respectively (Figure 1A). Similarly, we predicted 20524, 21871 and 21870 circRNAs in the lens from three WT mice and 2676, 
3638 and 3116 unique circRNAs in those mice. Analysis of the number of exons for these circRNAs revealed that the majority (78.29\%) of circRNAs had less than seven exons (Figure 1B). With standard metrics, we detected the length distribution of circRNAs. Apparently, the majority of circRNAs had a length of $>200 \mathrm{nt}$ with a median length of 801-900 nt in a wide range (Figure 1C). Analysis of their locations indicated that these circRNAs were widely localized in all chromosomes and the mitochondrial genome and the great numbers of circRNAs occurred on chromosome 2 while the fewest numbers of circRNAs in $Y$ chromosome and the mitochondrial genome (two circRNAs for each) (Figure 1D).

\section{Characteristics of differentially expressed circRNA profiles}

Aligned the circRNA database, we identified 49494 circRNA candidates ( $\geq 2$ back-spliced reads) and 40228 novel circRNAs (Figure 2A). Analysis of the secondary sequences indicated that $92.44 \%$ of reads in 49494 circRNA candidates contained the sense-overlapping circRNAs (Figure 2B). Compared with the WT lens, 149 circRNA transcripts were significantly elevated and 172 reduced in the lens from $\mathrm{Crybb2^{-/ }}$ mice (Figure 2C-D).

\section{Establishment of a circRNA-miRNA network}

CircRNA can sponge miRNA to enhance the expression of mRNAs the miRNA targets and one circRNA can bind to multiple miRNAs. To explore the potential functions of circrNAs, we employed the Miranda software to predict the interaction of circRNAs with miRNAs. After extracted top 300 circRNA-miRNA interaction pairs, we generated a network map of circRNA-miRNA interactions (Figure 3).

\section{Functional analysis of differentially expressed circRNAs}

To better understand the functions of the differentially expressed circRNAs, we analyzed the functions of their target genes using GO and the potential pathways they were involved by the KEGG pathway. The results indicated that those circRNAs participated in various biological processes, such as lens fiber cell development, calcium channel complex, structural constituent of the lens (Figure 4A). The KEGG analysis revealed that those differentially expressed circRNAs were enriched in several vital pathways, such as the canonical Wnt signaling pathway (Figure 4B)

\section{Validation of differentially expressed circRNAs in the lens.}

In comparison with that from RNA-seq (Figure 5A), we quantified eight differentially expressed circRNAs by RT-PCR and found that they had similar expression patterns in (Figure 5B).

\section{Discussion}

CircRNAs can regulate the pathological process of various types of diseases, including ocular diseases[11-13]. However, the role of circRNAs in cataract is rarely reported, particularly in the regulation of Crybb2 knockout on circRNA expression in the lens. In this study, we identified 49,494 circRNAs in mouse lens and 321 differentially expressed circRNAs in the lens of $\mathrm{Crybb2}^{--}$mice, including 149 
upregulated and 172 downregulated, related to that in the WT mice. We validated eight differentially expressed circRNAs by qRT-PCR. Such novel data clearly demonstrated that Crybb2 deficiency remarkably modulated the expression profiles of circRNAs in the lens of mice.

It is well known that circRNA can functionally sponge miRNAs to abrogate their inhibition on mRNA translation, regulating cell proliferation, differentiation, and apoptosis. Based on prediction, we constructed the differentially expressed circRNA-miRNA interaction network. This network suggests that some circRNAs may interact with several miRNAs, such as miR-125b and miR-326 that are expressed in the lens[14-15]. The results indicated the potential associations between circRNAs and their target genes, which provided an important reference for studying the interaction of other differentially expressed circRNAs and their potential targets. For example, circRNA-36601 is located in the Crybb2, and its rs78378222 polymorphism in the 3'-untranslated region of TP53 mRNA can modify the miR-125binduced apoptosis of lens epithelial cells, leading to the development of aging-associated cataracts[16]. The circRNA-02231 may target miR-25b. In addition, circRNA-14121 or circRNA-16505 may target miR326, which inhibits CRYBB2 expression by down-regulating FGF1 because miR-326 antagomer enhances CRYBB2 expression[15]. Hence, induction of circRNA-14121 or circRNA-16505 expression, like miR-326 antagomar, may be promising candidates to inhibit the progression of age-related cataract. Therefore, this network, if validated, may uncover the regulation of CRYBB2 expression and therapeutic targets for intervention of cataract.

Further Gene Ontology and KEGG analysis indicated that those differentially expressed circRNAs might be involved in various biological processes, such as lens fiber cell development, calcium channel complex, structural constituent of the lens. Those differentially expressed circRNAs also participated in the functional pathways, such as the canonical Wnt signal pathway, autophagy and others. Thus, those differentially expressed circRNAs are important for the lens development and health in mice. We are interested in further investigating the molecular mechanisms by which those differentially expressed circRNAs regulate the development and progression of cataract.

Our study has two limitations. This study had a relatively smaller sample size and lack of information on the development and severity of cataract in mice. In addition, this study was only conducted in mice and further studies in human lens with a bigger sample size are necessary to validate the role of circRNAs in the development and progression of cataract.

In summary, we identified a great number of differentially expressed circRNAs in the lens of $\mathrm{Crybb2^{-1 }}$ mice, with which we constructed a circRNA-miRNA interaction network. Those differentially expressed circRNAs participated in various biological processes and were involved in functional pathways. Our findings may provide a valuable platform for identifying circRNAs that affect the lens development. Therefore, our findings may uncover valuable biomarkers for diagnosis and therapy for cataract.

\section{Declarations}




\section{Funding}

Not applicable.

\section{Conflicts of interest/Competing interests}

The authors declare that they have no competing interests.

\section{Ethics approval}

Not applicable.

\section{Consent to participate}

Not applicable.

\section{Consent for publication}

Not applicable.

\section{Availability of data and material}

RNA-seq data are available on Sequence Read Archive (SRA),PRJNA728889

(https://www.ncbi.nlm.nih.gov/sra/PRJNA728889)

\section{Code availability}

Not applicable.

\section{Authors' contributions}

QG and HX wrote the manuscript. YD and LC performed the bioinformatic analysis. ZN and YZ contributed to the acquisition and interpretation of data. QL revised the manuscript. All authors read and approved the final manuscript.

\section{ACKNOWLEDGMENTS}

The authors would like to thank Shanghai OE Biotech Co.,Ltd. (Shanghai, China) for assistance with bioinformatic analysis and Medjaden Bioscience Ltd. for assisting in the preparation of the manuscript.

\section{References}

1. Zhang J,Huang CG,Li WJ, et al(2006).Establishment of a $\beta B 2$ crystallin gene knockout mice model.Acad J Second Military Med Univ 27:1246-1249. 
2.Jun $\mathrm{Z}$,Jing L,Caiguo $\mathrm{H}$,et al(2008).Targeted knockout of the mouse betaB2-crystallin gene (Crybb2) induces age-related cataract. Invest Ophthalmol Vis Sci 49:5476-5483.https://doi.org/10.1167/iovs.082179

3. Zhaoyong L, Chuan H, Chun B,et al(2015). Exon-intron circular RNAs regulate transcription in the nucleus. Nat Struct Mol Biol22:256-264. https://doi.org/10.1038/nsmb.2959

4. William W D, Weining Y, Elizabeth $L$, et al(2016). Foxo3 circular RNA retards cell cycle progression via forming ternary complexes with p21 and CDK2. Nucleic Acids Res 44:28462858.https://doi.org/10.1093/nar/gkw027

5. Thomas B. H, Trine I. J, Bettina H. C, et al(2013). Natural RNA circles function as efficient microRNA sponges.Nature 495:384-388.https://doi.org/10.1038/nature11993

6. Akhter,R(2018).Circular RNA and Alzheimer's disease. Adv Exp Med Biol 1087:239243.https://doi.org/10.1007/978-981-13-1426-1_19

7. Rui-Chao L, Shun K, Fan-Kai M,et al(2018).CiRS-7 promotes growth and metastasis of esophageal squamous cell carcinoma via regulation of miR-7/HOXB13.Cell Death Dis 9:838. https://doi.org/10.1038/s41419-018-0852-y

8. Luo,Y.Q, Liu,S.Y,Yao,K(2017). Transcriptome-wide investigation of mRNA/circRNA in miR-184 and its r.57c > u mutant type treatment of human lens epithelial cells. Mol Ther Nucleic Acids 7:71-80. https://doi.org/10.1016/j.omtn.2017.02.008

9. Xin L, Baihui L, Menglong Z,et al(2018).Circular RNA HIPK ${ }_{3}$ regulates human lens epithelial cells proliferation and apoptosis by targeting the miR-193a/CRYAA axis.Biochem Biophys Res Commun.503:2277-2285.https://doi.org/10.1016/j.bbrc.2018.06.149

10. CaiXia L, XiaoMei S, Na L,et al(2018). CircRNAs in the tree shrew (Tupaia belangeri) brain during postnatal development and aging.Aging (Albany NY)10:833-852. https://doi.org/10.18632/aging.101437

11. Lei, M. Zheng, G.T. Ning,Q.Q,et al(2020).Translation and functional roles of circular RNAs in human cancer.Mol Cancer 19:30. https://doi.org/10.1186/s12943-020-1135-7

12. Qu,X.L. Li, Z.X. Chen, J,et al(2020).The emerging roles of circular RNAs in CNS injuries.J Neurosci Res 98:1485-1497.https://doi.org/10.1002/jnr.24591

13. Jinghua W, Shushan $Y$, Jinghan $Y$, et al(2020).Non-coding RNAs in rheumatoid arthritis: from bench to bedside.Front Immunol10:3129.https://doi.org/10.3389/fimmu.2019.03129

14.Yu Q, Jiangyue Z, Xiaojie M,et al(2014). MicroRNA-125b inhibits lens epithelial cell apoptosis by targeting p53 in age-related cataract. Biochim Biophys Acta 1842:2439-2447.

https://doi.org/10.1016/j.bbadis.2014.10.002 
15. Hanxiao R, Haibo T, Qian G.et al(2018). MiR-326 antagomir delays the progression of age-related cataract by upregulating FGF1-mediated expression of betaB2-crystallin. Biochem Biophys Res Commun 505:505-510. https://doi.org/10.1016/j.bbrc.2018.09.049

16.Yang Z, Xiao L, Siquan Z(2016).rs78378222 polymorphism in the 3'-untranslated region of TP53 contributes to development of age-associated cataracts by modifying microRNA-125b-induced apoptosis of lens epithelial cells.Mol Med Rep 14:2305-2310. https://doi.org/10.3892/mmr.2016.5465

\section{Figures}
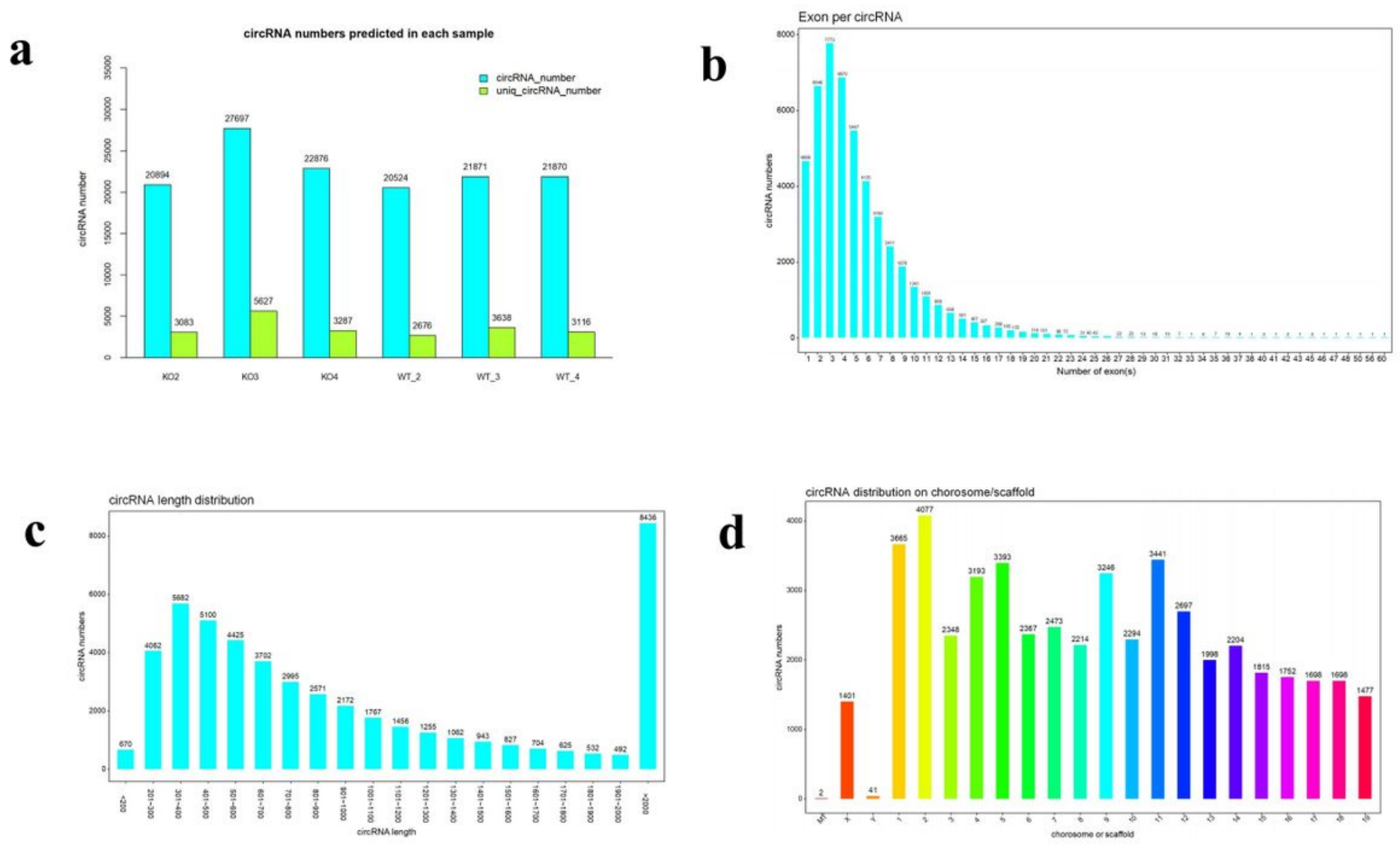

Figure 1

The characteristics of overall circRNAs. A. The numbers of circRNA predicted in each sample. B. The exon numbers of circRNAs in mouse lens. C. The circRNA length distribution. D. The distribution of circRNAs among chromosomes. 

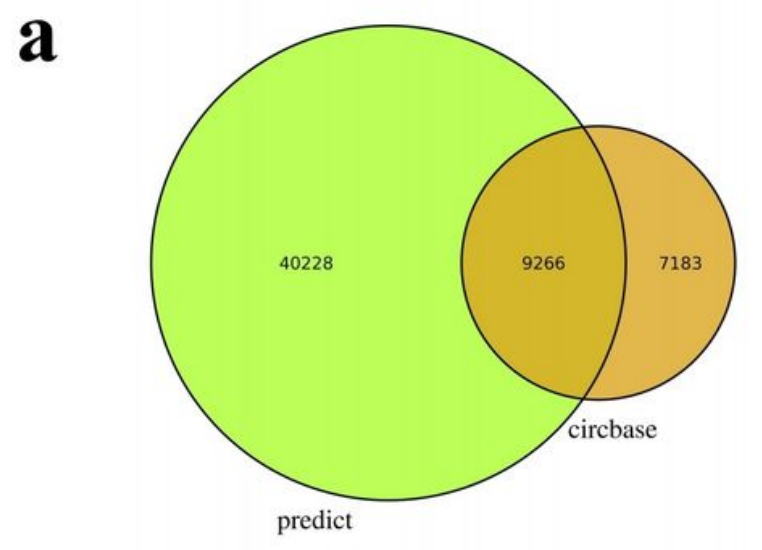

b
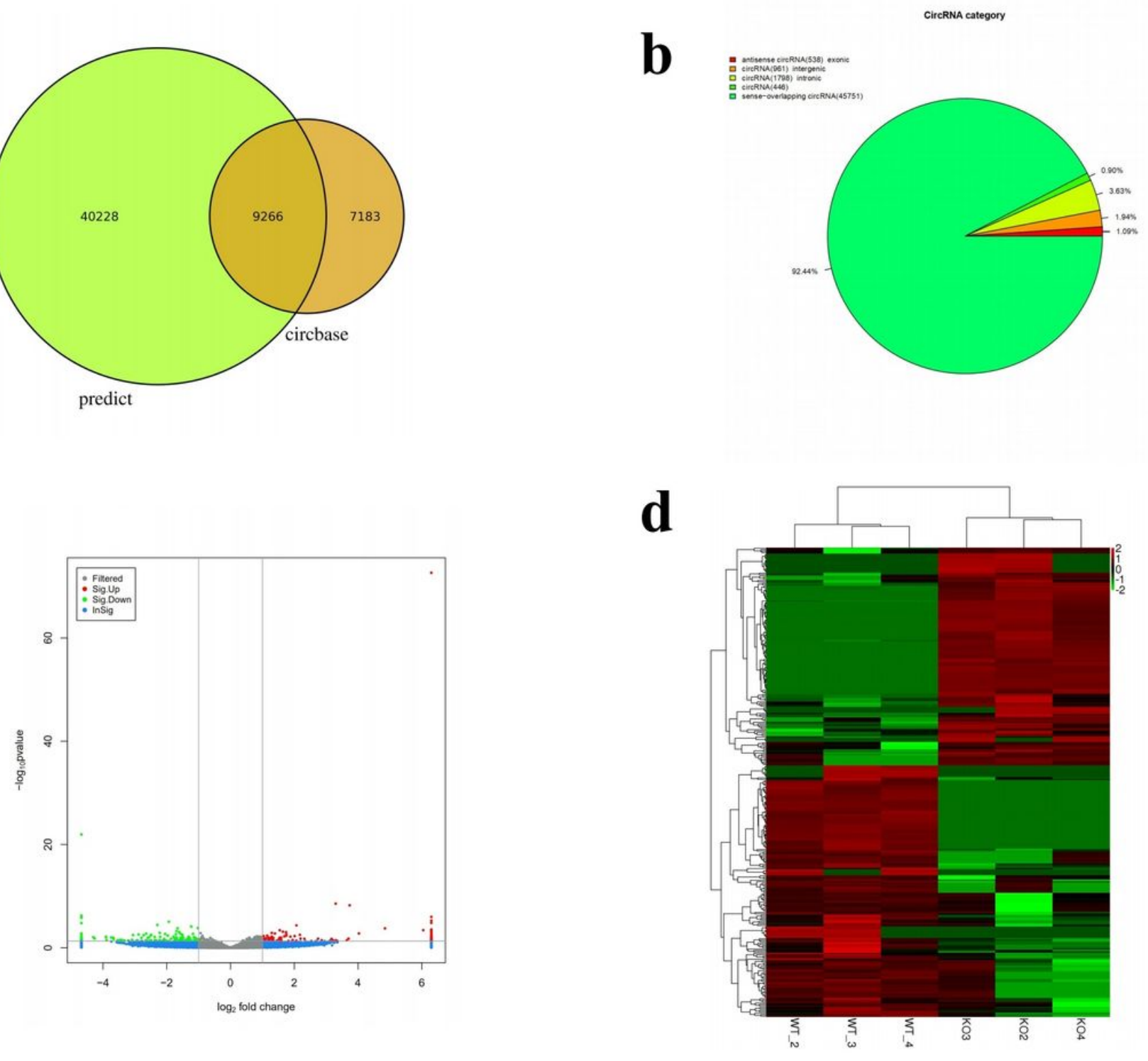

\section{Figure 2}

The differentially expressed circRNA profiles in the lens. A. The identified circRNAs compared with that in the database. B. The percentage of differentially expressed circRNAs in each genomic locus (senseoverlapping, intronic, intergenic, exonic, antisense). C. Volcano plot analysis of differentially expressed circRNAs. The $\mathrm{X}$-axis was the fold change expressed as log2, and the Y-axis was P-value expressed as $\log 10$. The gray dots represent circRNAs with no statistical significance between the WT and KO mice. The red dots in the plot represent the up-regulated circRNAs while the green are downregulated. The blue ones represent circRNAs with changes in 2.0-fold, but not statistically significant. D. The heatmap of differentially expressed circRNAs between the WT and KO mice. Red: The upregulated; Green: The downregulated. 


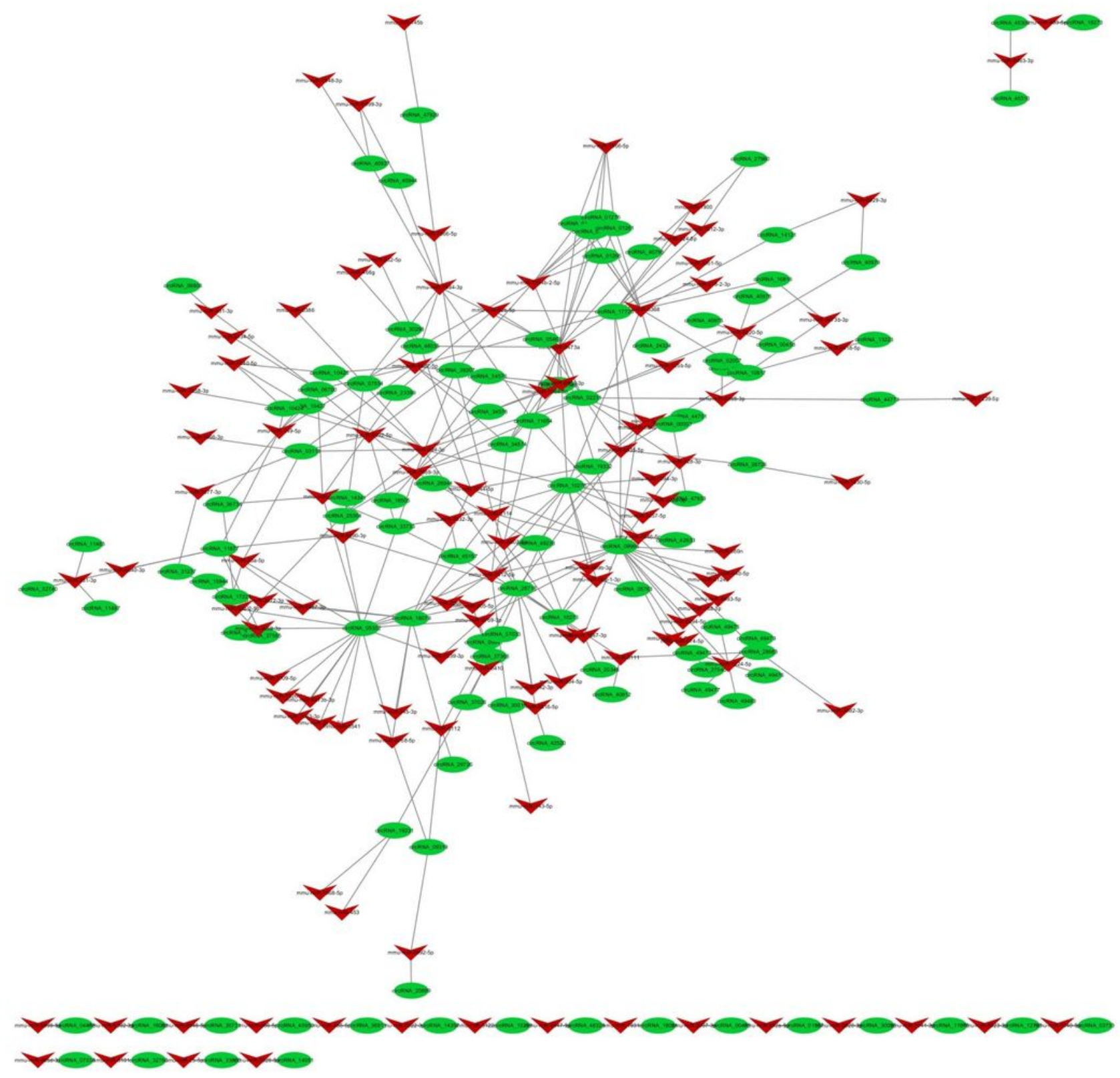

\section{Figure 3}

The circRNA-miRNA interaction network. The network map includes the differentially expressed circRNAs (green nodes) and their potential targeted miRNAs (red nodes). The edge denotes the relationship between circRNA and miRNA. 
a

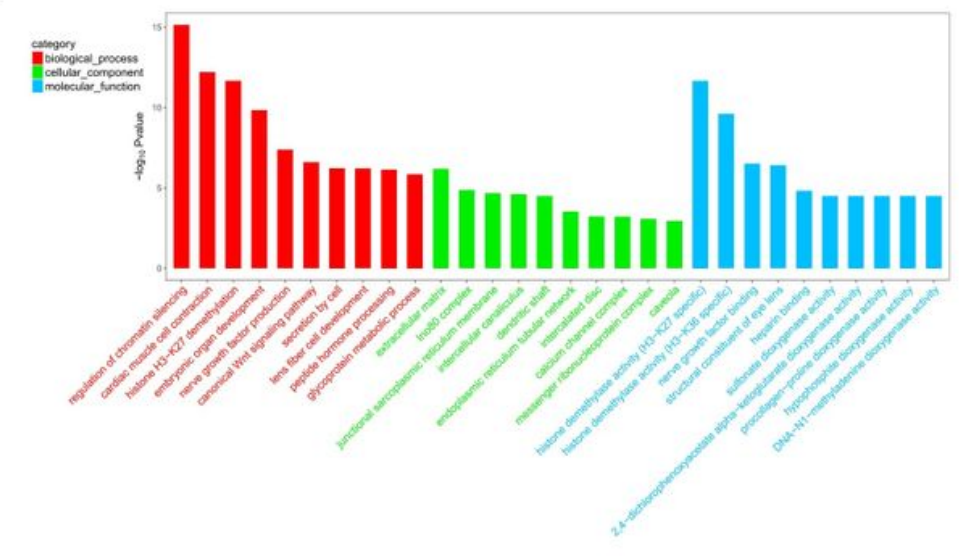

b

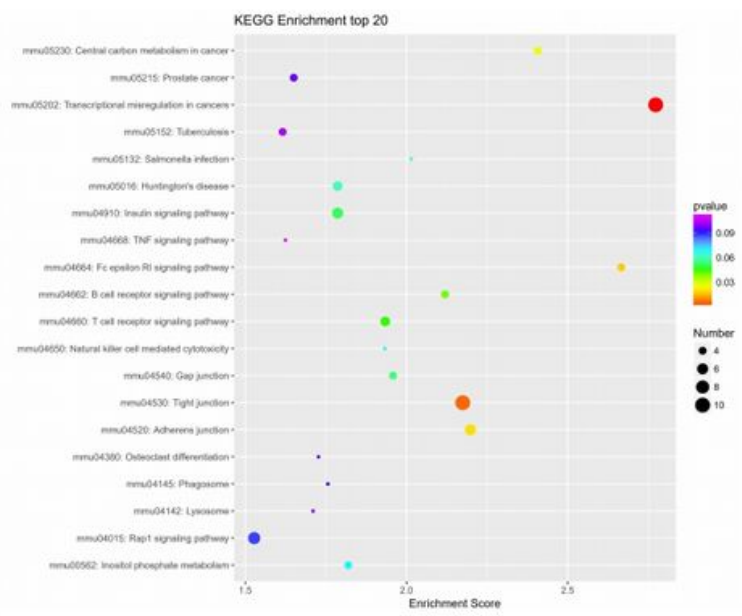

Figure 4

The gene ontology (GO) and KEGG analysis of the potential functions of differentially expressed circRNAs in the lens. A. GO analysis of the functional potentials of differentially expressed circRNAs in the lens. B. The KEGG analysis of the enrichment of differentially expressed circRNAs in the lens. Data are the top changed circRNAs. The $\mathrm{X}$-axis represents the rich factors (ratio of the enriched differential gene in each pathway). The Y-axis shows the pathway name. The bubble represents the enrichment number of differential genes. The $\mathrm{p}$-values are indicated by different colors from green to purple.

a

b
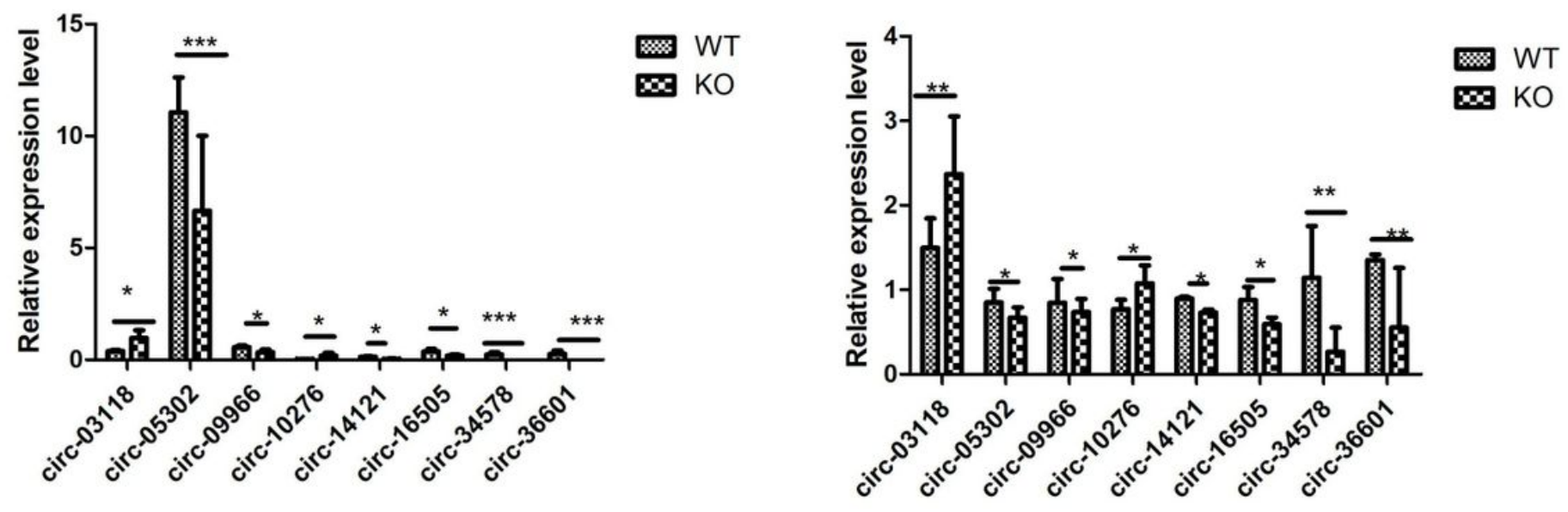

Figure 5

Quantitative RT-PCR validates the differentially expressed circRNAs in the lens. A. RNA-seq analysis of the differentially expressed circRNAs. B. Quantitative RT-PCR analysis of the differentially expressed 
circRNAs. Data are expressed as the mean \pm SD of each group from three separate experiments. ${ }^{*}<0.05$, $\star \star P<0.01, * \star * P<0.001$ determined by Student $T$ test. 